Scientific Paper

\title{
Effect of electrode contact area on the information content of the recorded electrogastrograms: An analysis based on Rényi entropy and Teager-Kaiser Energy
}

\author{
Paramasivam ALAGUMARIAPPAN ${ }^{1}$, Kamalanand KRISHNAMURTHY ${ }^{1, a}$, Sundravadivelu KANDIAH ${ }^{2}$, Mannar Jawahar \\ PONNUSWAMY ${ }^{3}$ \\ ${ }^{1}$ Department of Instrumentation Engineering, MIT Campus, Anna University, Chennai 600044, India \\ ${ }^{2}$ Shree Balaji Clinic, Ezhil Nagar, Selaiyur, Chennai 600073, India \\ ${ }^{3}$ Anna University, Chennai 600025, India \\ ${ }^{a}$ E-mail address: kkamalresearch@gmail.com,kamalanand@mitindia.edu
}

(received 2 March 2017; revised 18 April 2017; accepted 2 May 2017)

\begin{abstract}
Electrogastrograms (EGG) are electrical signals originating from the digestive system, which are closely correlated with its mechanical activity. Electrogastrography is an efficient non-invasive method for examining the physiological and pathological states of the human digestive system. There are several factors such as fat conductivity, abdominal thickness, change in electrode surface area etc, which affects the quality of the recorded EGG signals. In this work, the effect of variations in the contact area of surface electrodes on the information content of the measured electrogastrograms is analyzed using Rényi entropy and Teager-Kaiser Energy (TKE). Two different circular cutaneous electrodes with approximate contact areas of $201.14 \mathrm{~mm}^{2}$ and $283.64 \mathrm{~mm}^{2}$, have been adopted and EGG signals were acquired using the standard three electrode protocol. Further, the information content of the measured EGG signals were analyzed using the computed values of entropy and energy. Results demonstrate that the information content of the measured EGG signals increases by $6.72 \%$ for an increase in the contact area of the surface electrode by $29.09 \%$. Further, it was observed that the average energy increases with increase in the contact surface area. This work appears to be of high clinical significance since the accurate measurement of EGG signals without loss in its information content, is highly useful for the design of diagnostic assistance tools for automated diagnosis and mass screening of digestive disorders.
\end{abstract}

Key words: electrogastrography; biopotentials; cutaneous electrodes; Rényi entropy; Teager-Kaiser energy operator; information content.

\section{Introduction}

The gastrointestinal (GI) tract is a tubular system that begins with the mouth starting from oropharynx, esophagus, stomach, small and large intestines and terminating at the anus. Process of digestion has several stages in which food is broken down and absorbed [1]. Additionally, the gastrointestinal tract is a route for drug administration, absorption, biotransformation, detoxification, and excretion [2]. The electrophysiology of the digestive system is highly complex and the electrical activity of the digestive system is closely correlated with the mechanical process of digestion [3].

Electrogastrography is the measurement and recording of the electrical activity associated with the process of digestion. In this technique, the electrical signals originating from the abdomen over the stomach are recorded using cutaneous electrodes placed on the abdominal skin [4]. The recorded signal is called an electrogastrogram and is clinically useful for diagnosis of various digestive disorders such as Gastroesophageal reflux diesaese, functional dyspepsia, stomach ulcer, etc. Further, the non-invasive nature of this method makes it a simple, cost effective and efficient technique for classification of the pathological and physiological states of the digestive system [5].

The complexity of the physiological system and the associated information content can be quantified using a number of techniques derived from the fields of nonlinear dynamics, thermodynamics and statistical mechanics [6]. The entropy associated with the system is derived from the second law of thermodynamics and has found its application in biomedical signals and time series analysis. The entropy of a system is a measure of the disorder associated with the system and hence is used to quantify the information content of a signal. In recent years, several researchers have used entropy measures for solving significant problems in the field of biomedical engineering [7-10]. Bromiley et al. (2004) [11] 
have presented the utility of entropy measures such as Shannon entropy and Rényi entropy. Further, the authors have derived the correlation between Rényi entropy and information content. Also, the authors have concluded that Rényi entropy is a monotonic function of the information content and can be interchangeably used.

Energy of the system is defined as the capacity of a system to do work. Biological systems are natural energy transformers. The activity of biological systems produces associated electric signals which are closely related with the system dynamics [12]. Teager-Kaiser Energy operator is an efficient tool for analyzing the energy of biosignals in both continuous and discrete domains. The Teager-Kaiser Energy is a measure of the energy required by the physiological system to produce the associated biosignal [13].

The change in electrode conductive area affects the quality of the electrophysiological measurements. Several biomedical researchers have presented the effects of different electrode surface area for analysis of biosignals [14-16]. Mintchev et al. (1997) [16] have experimentally concluded that the quality of the EGG signals depends on the electrode surface area used. Further, the authors have experimentally demonstrated that the specificity of the EGG test significantly varies with changes in the contact area of the surface electrodes used for measurement. Additionally, there are several other factors such as fat conductivity, abdominal thickness etc. affects the EGG signal recordings. Mintchev et al. (1998) [17] have presented the effect of different abdominal thickness on non invasive recordings of Electrogastrograms. Kim et al. (2012) [18] have experimented two complementary methods such as Magnetogastrograms and Electrogastrograms for measurement of gastric electric potentials. Further, the authors have quantified the effects of fat conductivity and thickness parameters on both electric and magnetic fields. Also, the authors have concluded that the increase in thickness of fat layer merely affects the amplitude of the EGG signal.

The objective of this work is to analyze the effect of electrode surface area on the information content of the measured electrogastrograms.

\section{Methodology}

\section{EGG Signal Acquisition}

In this work, a three electrode EGG measurement system was adopted for noninvasive measurement of EGG signals. According to the standard electrode placement protocol, the first and second electrodes positioned below the left costal margin and, between the xyphoid process and the umbilicus, respectively. The third cutaneous electrode is placed away from the stomach region for isolation purpose [19, 20]. Two different conductive solid gel $\mathrm{Ag} / \mathrm{AgCl}$ surface electrodes with contact areas of $201.14 \mathrm{~mm}_{2}$ and $283.64 \mathrm{~mm}_{2}$ were used to record EGG signals from ten normal individuals in consecutive sessions. The recordings were used to analyze the effect of the change in electrode contact areas on the information content of
EGG signals. Further, the acquired EGG signals were amplified using an instrumentation amplifier designed using IC AD624. The amplified EGG signals were acquired using LABVIEW data acquisition card (NI USB-6009) and recorded using LABVIEW (V 14.0.1) software. The EGG signals of ten normal male adults were recorded with their consent, for a period of 10 minutes using the two different electrodes with varied contact surface area. A sampling time of 0.01 seconds was used for recording the EGG signals. Further, the EGG signals were de-trended and were further analyzed.

\section{Rényi entropy}

The statistical concept of order and disorder was revealed by the investigations of Boltzmann and Gibbs in statistical physics. The quantitative connection is expressed by [21]:

entropy $=k \log D$

Eq. 1

where, $k$ is the Boltzmann constant, and $D$ is a quantitative measure of the system disorder. In natural system, entropy appears to be a positive function of time and tends to reach a state of maximum entropy [21].

The concept of entropy was introduced by Rudolph Clausius in connection with the second law of thermodynamics [6]. Entropy is a measure of disorder associated with a system and hence is a measure of information content, uncertainty and complexity of the system [22]. The concept of entropy is utilized in various fields of science and engineering such as statistical mechanics, information theory, biomedical signal processing, chaos theory, neural networks, mathematical linguistics, and taxonomy [6]. Alfred Rényi proposed the most general definition of information measure that would preserve the additivity for independent events and was compatible with probability axioms [23].

For a given a sample of probabilities $p_{i \text { : }}$

$\sum_{i=1}^{N} p_{i}=1$

Rényi entropy of the sample $H(\alpha)$ is given by [22]:

$H(\alpha)=\frac{1}{1-\alpha} \ln \left(\sum_{i=1}^{n} p_{i}^{\alpha}\right)$

where $p_{i}$ is the probability that a random variable takes a given value out of $n$ values, and $\alpha$ is the order of the entropy measure. As $\alpha$ increases, the entropy values become more sensitive to higher probabilities and less sensitive to lower probabilities. The Rényi entropy is a monotonic function of the information which implies that the Rényi entropy and information content can be used interchangeably in any practical applications [11]. The information content of the recorded EGG signals for variations in the contact surface area of the measuring electrodes was quantified using the Rényi entropy.

\section{Teager-Kaiser Energy operator}

The energy of a simple oscillation is proportional to both the square of the amplitude and the square of the frequency of the oscillation. Teager-Kaiser energy (TKE) operator is an efficient 
tool for analyzing both continuous and discrete signals from the energy point of view [24].

The discrete Teager-Kaiser energy operator $\Psi$ is defined as [24]:

$\Psi[x(n)]=x^{2}(n)-x(n+1) \cdot x(n-1)$

Eq. 4

where, $x$ is the measured EGG value and $n$ is the sample number [25]. In recent years, the Teager-Kaiser energy operator is used to calculate the overall change in energy of the signal on biological systems [26]. Further, the Teager-Kaiser Energy operator tries to model the energy of the source of the signal, and not the energy of the signal which is actually measured [13]. In this work, the energy associated with the measured EGG signals was quantified using the TKE operator.

\section{Results and Discussion}

Figures 1a and 1b show a typical EGG signal measured using the surface electrode with a contact area of $201.14 \mathrm{~mm}^{2}$, for a period of 10 minutes and the single sided amplitude spectrum of the signal, respectively. It is observed that the frequency range of 0.01 to $0.1 \mathrm{~Hz}$ is prominent in the FFT of the EGG signals acquired using the surface electrode with a contact area of $201.14 \mathrm{~mm}^{2}$.

Figures 2a and 2b show a typical EGG signal measured using the surface electrode with a contact area of $283.64 \mathrm{~mm}^{2}$, for a period of 10 minutes and the single sided amplitude spectrum of the signal, respectively. It is observed that the frequency range of 0.01 to $0.06 \mathrm{~Hz}$ is prominent in the FFT of the EGG signals acquired using the surface electrode with a contact area of $283.64 \mathrm{~mm}^{2}$. While comparing the single sided amplitude-frequency spectrum of the EGG signals acquired from two different electrodes, it is clearly observed that the contact surface area affects both the amplitude and frequency of the EGG signal.

Figures 3a-e show the average Rényi entropy of normalized EGG signals for two different contact area of measuring electrodes, for five different $\alpha$ values ( $\alpha=0.2, \alpha=0.4, \alpha=0.6$, $\alpha=0.8$ and $\alpha=0.9$ ) respectively. It is seen that the average Rényi entropy increases with increase in contact surface area. This phenomenon may relate to the increased signal collecting ability of the electrode with larger surface area. Further, it is observed that the Rényi entropy increases with increase in $\alpha$. Hence at higher orders, the relation between the Rényi Entropy and Surface area is more prominent. Since, Rényi entropy is a measure of the information content in the measured EGG signals, it is demonstrated that the information content of the EGG signals increases with increase in the contact area of the surface electrodes used for acquiring the signals. Also, the difference in the Renyi entropy values of EGG signals acquired from electrodes with different contact areas, was found to be statistically significant $(\mathrm{P}<0.006)$.

While comparing the percentage change in the information content (with $=0.9$ ) with a change in the contact area of the measuring surface electrode, it is found that an increase of $29.1 \%$ in electrode surface area leads to an increase in the information content of the measured EGG signals by $6.72 \%$. a)

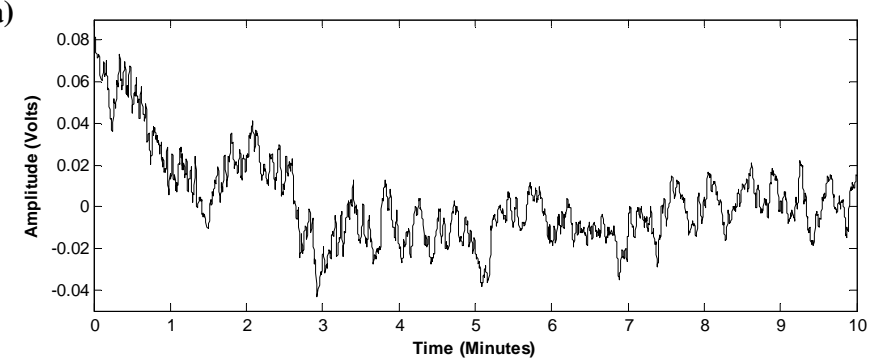

b)

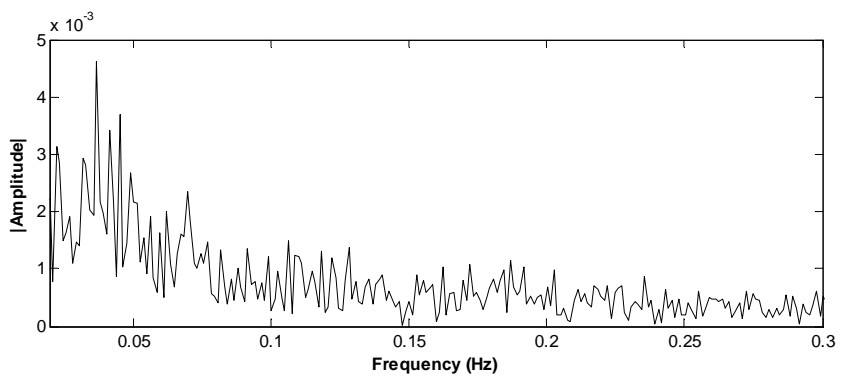

Figure 1. (a) A typical EGG signal measured using the surface electrode with a contact area of $201.14 \mathbf{m m}^{2}$, (b) the single sided amplitude spectrum of the EGG signal.

a)

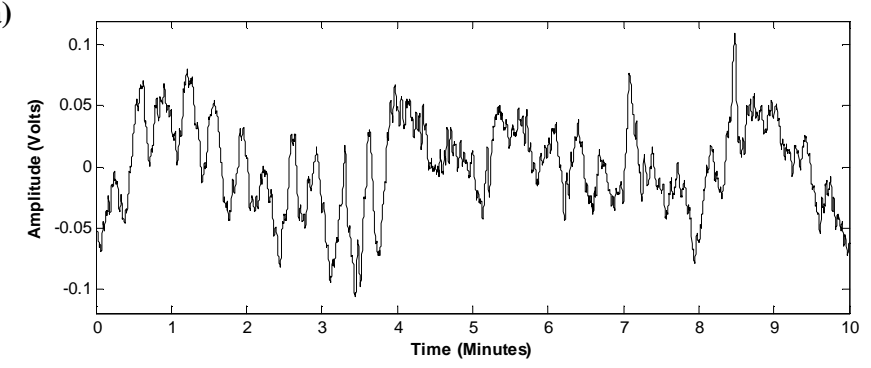

b)

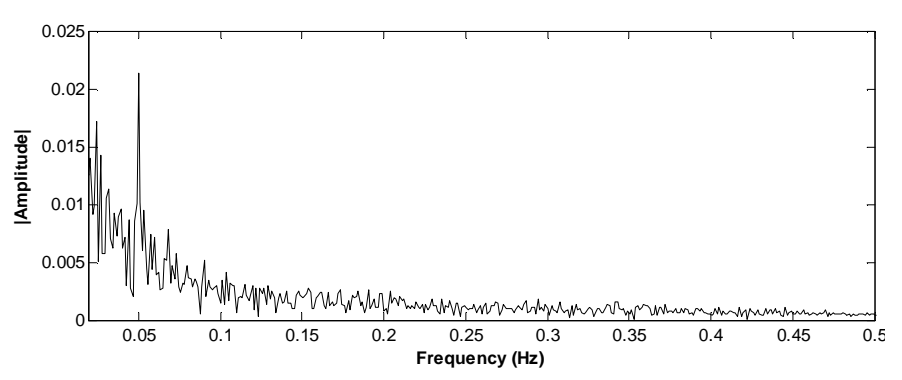

Figure 2. (a) A typical EGG signal measured using the surface electrode with a contact area of $283.64 \mathbf{m m}^{2}$, (b) the single sided amplitude spectrum of the EGG signal. 
a)

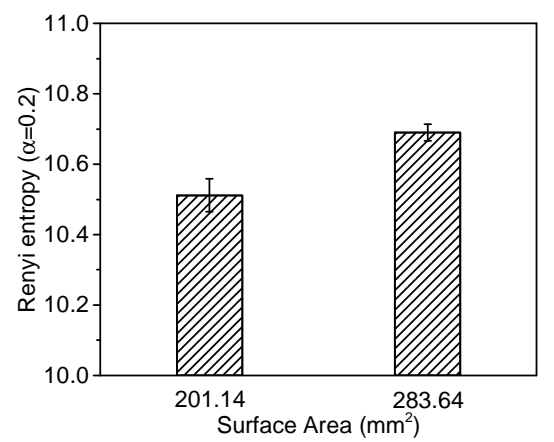

b)

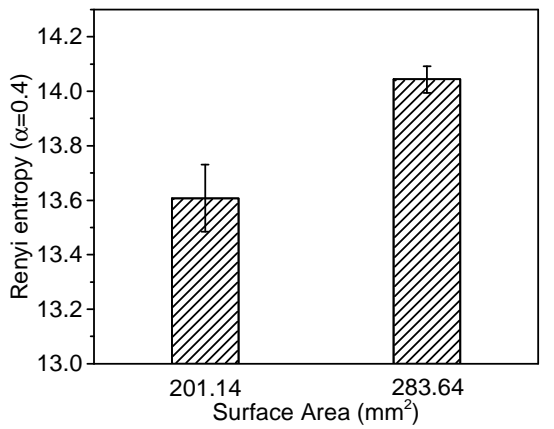

c)

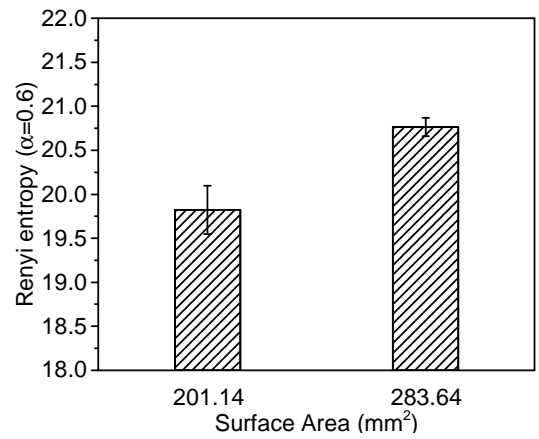

d)

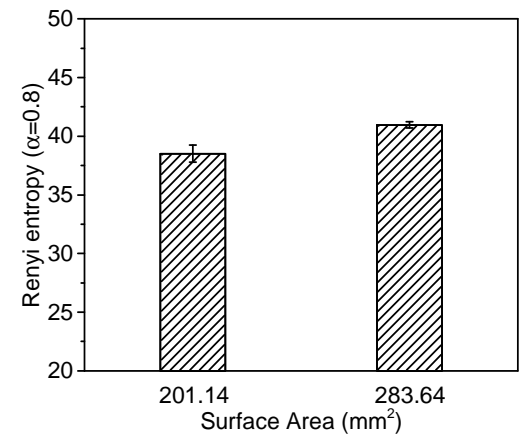

e)

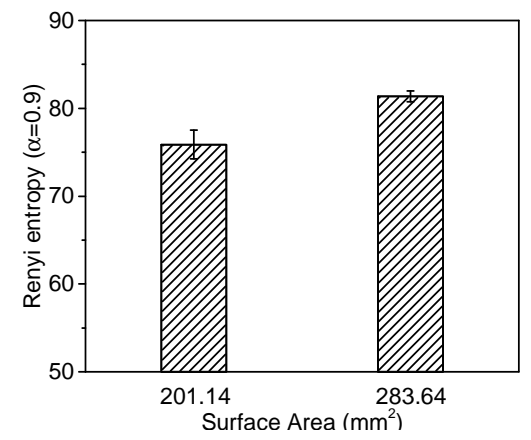

Figure 3. The Rényi entropy (Mean \pm Standard Error) of EGG signals collected from electrodes with different contact area for five different $\alpha$ values (a) $\alpha=0.2$ (b) $\alpha=0.4$ (c) $\alpha=0.6$ (d) $\alpha=0.8$ and (e) $\alpha=0.9$.

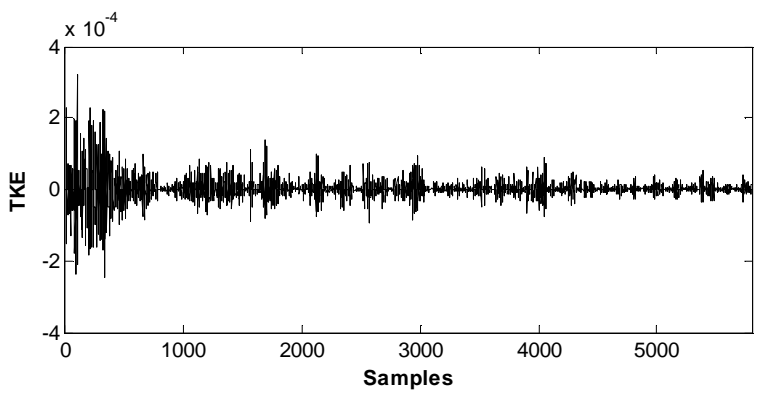

Figure 4. Teager-Kaiser Energy of the EGG signal acquired using surface electrode with $201.14 \mathrm{~mm}^{2}$ contact area as a function of sample number.

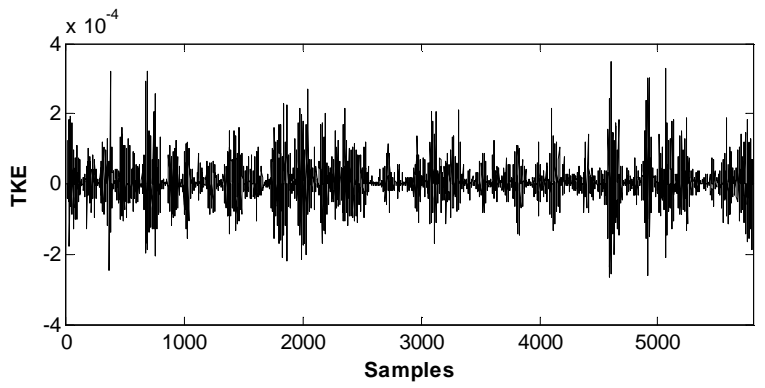

Figure 5. Teager-Kaiser Energy of the EGG signal acquired using surface electrode with $283.64 \mathrm{~mm}^{2}$ contact area as a function of sample number.

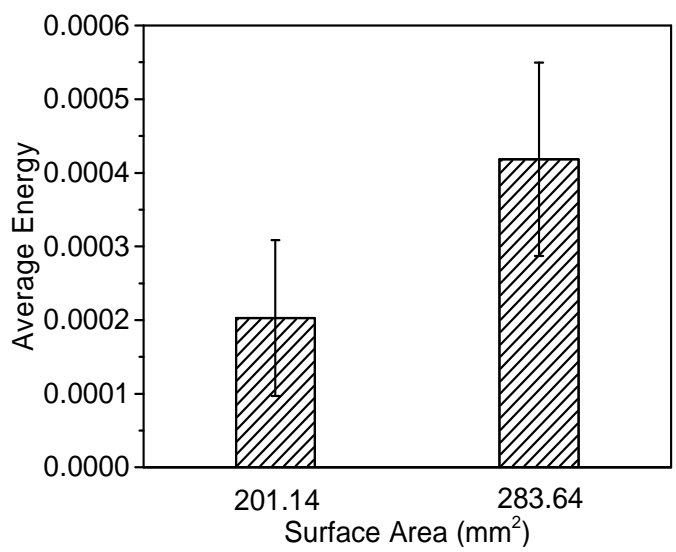

Figure 6. The Teager-Kaiser Energy (Mean \pm Standard Error) of EGG signals for different electrode contact areas.

Figure 4 and Figure 5 show the Teager-Kaiser Energy of the EGG signal acquired using the surface electrode with contact areas of $201.14 \mathrm{~mm}^{2}$ and $283.64 \mathrm{~mm}^{2}$, respectively as a function of sample number. It is observed that the TeagerKaiser Energy of the measured EGG signals assumes both positive and negative values. This phenomenon appears due to the fact that the Teager-Kaiser Energy operator tries to model the energy of the source of the signal rather than the energy of the signal itself [13]. This result demonstrates that the EGG signal is generated by multiple sources in the digestive system.

Hence, it is seen that the EGG signal consists of information about several subsystems of the human digestive system and using proper analysis, the EGG signals acquired using the adopted protocol can be used for assessment of various parts of 
the human digestive system. Figure 6 shows the average Teager-Kaiser Energy of normalized EGG signals, acquired from electrodes with different contact area of $201.14 \mathrm{~mm}^{2}$ and $283.64 \mathrm{~mm}^{2}$ electrode. It is seen that the average energy increases with increase in contact surface area.

\section{Conclusion}

The electrical signals originating from the digestive system are collectively called as Electrogastrograms. Electrogastrography is a cutaneous measurement and recording technique of the gastric electrical activity associated with the process of digestion [22]. Further, the non-invasive nature of this method and the mechano-electric correlations makes it a simple and efficient technique for classification of various disorders associated with the digestive system. The change in electrode surface area impacts the quality of the EGG recordings. In this work, the effect of variations in the contact area of surface electrodes on the information content of the measured electrogastrograms is analyzed using Rényi entropy and Teager-Kaiser Energy. The three electrode system and the standard recording protocol were adopted for recording the EGG signals. Two different circular cutaneous electrodes with approximate contact areas of $201.14 \mathrm{~mm}^{2}$ and $283.64 \mathrm{~mm}^{2}$ have been adopted. Further, the information content of the measured EGG signals was analyzed using the computed values of entropy and energy. Results demonstrate that the information content of the measured EGG signals increases by a factor of $6.72 \%$ for an increase in the contact area of the surface electrode by $29.1 \%$. Further, it was observed that the average energy increases with increase in the contact surface area and the contact surface area affects both the amplitude and frequency of the EGG signal. This work appears to be clinically significant since the measurement of EGG signals without loss in its information content is required for assessing the electromechanical associations and for the design of systems for diagnosis of digestive disorders.

\section{References}

[1] Swobodnik W, Soloway RS, Ditschuneit H (Eds). Gallstone disease: pathophysiology and therapeutic approaches. Springer Science \& Business Media, 2012.

[2] Siegel MA, Jacobson JJ, Braun RJ. Diseases of the Gastrointestinal Tract, Chapter 14. In: Burket's Oral Medicine: Diagnosis \& Treatment. Greenberg MS, Glick M (Eds). BC Decker, Inc. 2003;389-406.

[3] Neuman MR. The Electrical Engineering Handbook. Richard C. Dorf (Ed). CRC Press LLC, 2000.

[4] Yin J, Chen JD. Electrogastrography: methodology, validation and applications. Journal of Neurogastroenterology and Motility. 2013;19(1):5-17.

[5] Gopu G, Neelaveni R, Porkumaran K. Investigation of digestive system disorders using Electrogastrogram. Computer and Communication Engineering, 2008. ICCCE 2008. 201-205.

[6] Borowska M. Entropy-based algorithms in the analysis of biomedical signals. Studies in Logic, Grammar and Rhetoric. 2015;43(1):21-32.

[7] Rosso OA. Entropy changes in brain function. International Journal of Psychophysiology. 2007;64(1):75-80.

[8] Ahmad SA, Chappell PH. Moving approximate entropy applied to surface electromyographic signals. Biomedical Signal Processing and Control. 2008 Jan 31;3(1):88-93.

[9] Hassan M, Terrien J, Marque C, et al. Comparison between approximate entropy, correntropy and time reversibility: Application to uterine electromyogram signals. Medical Eng Phys. 2011;33(8):980-986.

[10] Sun R, Song R, Tong KY. Complexity analysis of EMG signals for patients after stroke during robot-aided rehabilitation training using fuzzy approximate entropy. IEEE Transactions on Neural Systems and Rehabilitation Engineering. 2014;22(5):1013-1019.

[11] Bromiley PA, Thacker NA, Bouhova-Thacker E. Shannon entropy, Rényi entropy, and information. Statistics and Inf. Series (2004004). 2004.

[12] Castner DG, Ratner BD. Biomedical surface science: Foundations to frontiers. Surface Science. 2002;500(1-3):28-60.

[13] Kvedalen E. Signal processing using the Teager energy operator and other nonlinear operators. Thesis. University of Oslo Department of Informatics. 2003.

[14] Kasicka-Jonderko A, Jonderko K, Krusiec-Swidergol B, et al. Comparison of multichannel electrogastrograms obtained with the use of three different electrode types. J Smooth Muscle Res. 2006;42(2-3):89-101.

[15] Taji B, Shirmohammadi S, Groza V, etc. Impact of skin-electrode interface on electrocardiogram measurements using conductive textile electrodes. IEEE Transactions on Instrumentation and Measurement. 2014;63(6):1412-1422.

[16] Mintchev M, Stickel A, Bowes K. Impact of different electrode surface areas on validity of human electrogastrograms. Medical and Biological Engineering and Computing. 1997;35(1):66-68.

[17] Mintchev MP, Bowes KL. Computer simulation of the effect of changing abdominal thickness on the electrogastrogram. Med Eng Phys. 1998;20(3):177-181. 
[18] Kim JH, Pullan AJ, Bradshaw LA, et al. Influence of body parameters on gastric bioelectric and biomagnetic fields in a realistic volume conductor. Physiol Meas. 2012;33(4):545-556.

[19] Riezzo G, Russo F, Indrio F. Electrogastrography in adults and children: the strength, pitfalls, and clinical significance of the cutaneous recording of the gastric electrical activity. Biomed Res Int. 2013;2013:282757.

[20] Parkman HP, Hasler WL, Barnett JL, et al. Electrogastrography: a document prepared by the gastric section of the American Motility Society Clinical GI Motility Testing Task Force. Neurogastroenterol Motil. 2003;15(2):89-102.

[21] Schrödinger E. What is life?: With mind and matter and autobiographical sketches. Cambridge University Press; 1992.

[22] Alagumariappan P, Rajagopal A, Krishnamurthy K. Complexity Analysis on Normal and Abnormal Electrogastrograms Using Tsallis Entropy. In: 3rd International Electronic and Flipped Conference on Entropy and Its Applications 2016. Multidisciplinary Digital Publishing Institute.

[23] Principe JC. Information theoretic learning: Rényi's entropy and kernel perspectives. Springer Science \& Business Media; 2010.

[24] Kaiser JF. On a simple algorithm to calculate the 'energy' of a signal. International Conference on Acoustics, Speech, and Signal Processing. Albuquerque, NM. 1990;(vol1):381-38.

[25] Solnik S, DeVita P, Rider P, et al. Teager-Kaiser Operator improves the accuracy of EMG onset detection independent of signal-tonoise ratio. Acta Bioeng Biomech. 2008;10(2):65-68.

[26] Lauer RT, Prosser LA. Use of the Teager-Kaiser energy operator for muscle activity detection in children. Ann Biomed Eng. 2009;37(8):1584-1593. 